\title{
Molecular dynamics modeling and simulation of void growth in two dimensions
}

\author{
H-J Chang ${ }^{1}$, J Segurado ${ }^{1,2}$, O Rodríguez de la Fuente ${ }^{3}$, B M Pabón ${ }^{3}$ and \\ J LLorca ${ }^{1,2}$
}

\begin{abstract}
The mechanisms of growth of a circular void by plastic deformation were studied by means of molecular dynamics in two dimensions (2D). While previous molecular dynamics (MD) simulations in three dimensions (3D) have been limited to small voids (up to $\approx 10 \mathrm{~nm}$ in radius), this strategy allows us to study the behavior of voids of up to $100 \mathrm{~nm}$ in radius. MD simulations showed that plastic deformation was triggered by the nucleation of dislocations at the atomic steps of the void surface in the whole range of void sizes studied. The yield stress, defined as stress necessary to nucleate stable dislocations, decreased with temperature, but the void growth rate was not very sensitive to this parameter. Simulations under uniaxial tension, uniaxial deformation and biaxial deformation showed that the void growth rate increased very rapidly with multiaxiality but it did not depend on the initial void radius. These results were compared with previous 3D MD and 2D dislocation dynamics simulations to establish a map of mechanisms and size effects for plastic void growth in crystalline solids.
\end{abstract}

(Some figures may appear in colour only in the online journal)

\section{Introduction}

Ductile failure of metallic materials is normally dictated by the growth of voids due to plastic deformation until fracture is triggered by the sudden formation of a macroscopic crack by coalescence of neighbor voids. The typical size of the voids found in metallic materials encompasses various orders of magnitude, from $\mathrm{nm}$ (as those associated with He bubbles created by neutron irradiation [1]) to $\mu \mathrm{m}$ (as those nucleated by the fracture or decohesion of brittle inclusions [2]) and the physical mechanisms of void growth depend on the size. For nm-sized voids, there is experimental evidence that void growth occurs by the emission of shear dislocation loops from the void surface, which evolve by cross slip to prismatic loops, 
leading to an increase in the void dimensions [3-7]. The critical stress to generate dislocation loops from the void surface is, however, very high and this mechanism is restricted to very high strain rates or low temperatures [8].

In the case of $\mu \mathrm{m}$-sized voids, it is well-established that void growth occurs as a result of plastic deformation in the bulk [9] and the mechanics of porous ductile solids has been extensively studied in the last decades, including the effect of multiaxiality, loading path, plastic anisotropy, matrix hardening, void shape, etc (see [9] for a comprehensive review on these topics). These analyses are based on standard plasticity models and thus do not take into account the influence of void size on void growth. However, void dimensions are of the order of the average spacing between mobile dislocations and dislocation sources in the case of micrometer-sized voids. Strong size effects are expected under these circumstances according to the results obtained for the plastic deformation of micrometer-sized wires in torsion, micropillars in compression or nanoindentation of thin films [10-12].

The analysis of size effects on void growth was initially carried out within the framework of strain gradient plasticity theories [13-17], which predicted that larger voids grew faster than smaller ones. The differences in growth rates as well as the critical size at which this size effect disappears were dependent on the length scale included in the strain gradient plasticity model. This was an important limitation of these theories to establish the regime at which size effects are relevant because the actual magnitude and physical significance of this parameter (or set of parameters) is not clear. More recently, the problem of void growth has been analyzed by means of 2D dislocation dynamics (DD) simulations [18-21]. These analyses demonstrated that larger voids grow faster than smaller ones and this behavior is triggered by the limited availability of dislocations/sources around the smaller voids. Void growth by plastic deformation is impaired by the absence of dislocation/sources around the smaller voids, but this limitation is not operative for larger voids [20]. As a consequence, the size effect on void growth disappears above a critical void radius, which is of the order of 1 to $2 \mu \mathrm{m}$ according to 2D DD simulations [20]. These results are in agreement with more recent 3D DD simulations of void growth [22].

The mechanisms of void nucleation in nm-sized voids have been studied by Lubarda $[5,23]$ using an ideal 2D dislocation model. He concluded that the critical stress for nucleation increased for very small voids but did not analyze void growth. Other authors used 3D atomistic simulations to study the nucleation and growth of nm-sized voids [24-32] and Potirniche et al [26] reported that smaller voids grew faster at this length scale, a size effect opposed to that found for micrometer-sized voids. However, atomistic simulations of voids growth were limited to very small voids (void radius was below $10 \mathrm{~nm}$ ) due to the huge computational costs associated with the simulation of larger voids and it is not clear whether the results reported by the analytical and atomistic simulations will hold for larger voids.

The main objective of this investigation was to understand the connection between the molecular dynamics (MD) simulations of nm-sized voids and the DD simulations of micrometer-size voids and this was achieved by means of MD of the growth of circular voids in 2D. To this end, a suitable interatomic potential was selected to obtain a 2D material whose behavior was in agreement with the standard results for dislocation plasticity in fec metals. Indeed, 2D atomistics simulations have limitations due to the influence of the 2D assumption on the material, dislocations and loading conditions. The 2D atomistic model only considered one layer of atoms and ignores the interactions between layers. Strains perpendicular to the 2D plane are neglected (as under plane strain conditions in 2D continuum models) and strain relaxation in the perpendicular direction is not possible. Under these circumstances, particular strain relaxation mechanisms may not be captured, as was the case presented by the 3D MD simulations of Gungor and Maroudas [33] for ultra-thin films which showed that the nucleation 
of a uniform dislocation network in the film was preferred to void growth under very large biaxial strain levels $(>8 \%)$. Another limitation is the point character of the defects generated in 2D. The interactions between dislocations in 2D are much simpler than in $3 \mathrm{D}$ (entanglements, jogs, etc.) and cannot be accounted for in the 2D model. However, it should be noted that our results mostly deal with the very first stages of plasticity, where interactions among dislocations are not as relevant. Nevertheless, most of these limitations can also be applied to 2D DD, which has been a very useful tool to study size effects in plasticity [34-39].

Bearing in mind these limitations for $2 \mathrm{D}$, simulations were carried out to understand the effect of temperature and multiaxiality on the yield stress defined as stress necessary to nucleate stable dislocations from the void surface and on the kinetics of void growth. They were compared with previous results of 3D MD and 2D DD simulations to establish a map of mechanisms and size effects for void growth.

\section{A 2D model material}

\subsection{MD model for a two-dimensional system}

The simulation of an atomistic system in 2D, showing both ductility and structural stability under external stresses, is not as direct and evident as in 3D and special care has to be taken in the correct choice of the interatomic potential. For instance, Abraham et al [40] reported that a 2D solid described by a pair potential of the Lennard-Jones (LJ) type is brittle while it shows significant dislocation activity prior to crack nucleation in 3D [41]. Our first attempts were in agreement with these observations and showed that a voided 2D solid described by a pair potential of the LJ type did not show a regular and continuous emission of dislocations: the 2D solid presented brittle fracture by the nucleation and growth of cracks from the void surface before any dislocations were emitted.

In order to overcome this limitation, the interaction between atoms in $2 \mathrm{D}$ was modeled using a 2D glue potential adapted from [42], and optimized for six nearest neighbors. The total energy of the system, $E_{\mathrm{tot}}$, composed by $N$ atoms is given by

$$
E_{\mathrm{tot}}=\frac{1}{2} \sum_{i, j}^{N} \Phi\left(r_{i j}\right)+\sum_{i}^{N} F\left(\rho_{i}\right)-A \sum_{i, j}^{n n} \cos \left(6 \theta_{i j}\right) .
$$

The first term $\Phi\left(r_{i j}\right)$ is a pair interaction between metal ions $i$ and $j$, which represents an effective electrostatic interaction and depends on their separation $r_{i j}$. The second part is a many-body term, which represents the energy $F\left(\rho_{i}\right)$ of ion $i$ for being embedded in a free electron sea, which in turn depends on the electronic density $\rho_{i}$ generated by the rest of the atoms at the position of ion $i$ according to

$$
\rho_{i}=\sum_{j}^{N} \rho\left(r_{i j}\right) .
$$

This term accounts for the metallic character of the 2D solid, and it has to be included to simulate plastic events, i.e., the nucleation of dislocations and their movement along slip planes.

All these functions are empirically constructed and were fitted to high order polynomials to reproduce accurately several physical properties of $\mathrm{Au}$, namely the lattice parameter, cohesion energy, the surface energies, the bulk modulus and the transverse phonon frequency at the $\mathrm{X}$-point of the Brillouin zone. Furthermore, the potential reproduces reasonably well vacancy formation energies, the thermal expansion coefficient and the melting temperature of $\mathrm{Au}$. The 
actual parameterizations can be found in [42]. In addition, this potential is, to the authors' knowledge, the only one able to reproduce the three different reconstructions of the ( 1111$)$, (1 110$)$ and (1 00 ) orientations of gold, rather distinctive of noble metals. The underlying point here is that this potential accurately takes into account the need of undercoordinated atoms to find an extra coordination, which is probably important for dislocation nucleation through the collapse of surface steps, where the surface atoms are particularly undercoordinated.

It was also found that the 2D solid was unstable if the total energy only included the first two terms in equation (1). Localized regions of the solid rotated under the application of an external stress leading to the formation of a polycrystal. To avoid this problem, a third angular term was added to the interatomic potential, which avoids local rotations of the solid under tension. Every atom $i$ has an additional contribution to the potential energy, where the sum extended to all $n n$ nearest neighbors. $\theta_{i j}$ is the angle formed between the vector connecting atoms $i$ and $j$ and the $x$-axis of the simulation cell, which defines one of the three compact directions of the hexagonal lattice. $A$ is a constant whose value was $0.01 \mathrm{eV}$.

In the MD model, the force between any pair of two atoms $i$ and $j$ is derived from total potential energy $\left(E_{\text {tot }}\right)$ according to

$$
f^{i j}=\frac{\partial E_{\text {tot }}}{\partial r^{i j}}
$$

and the dipole force tensor at each atom $i$ is given by

$$
\boldsymbol{\beta}^{i}=\frac{1}{\Omega^{i}}\left[\sum_{j \neq i}^{N_{n}}\left(f^{i j} \otimes r^{i j}\right)-m^{i} \boldsymbol{v}^{i} \otimes \boldsymbol{v}^{i}\right],
$$

where $N_{n}$ is the number of nearest neighbor atoms, $\Omega^{i}$ is the atomic volume and $m^{i}$ and $\boldsymbol{v}^{i}$ are the mass $i$ and the velocity of atom $i$, respectively. The stress for the system of atoms is defined as a volume average of the dipole force tensor

$$
\sigma=\frac{1}{N^{*}} \sum_{i}^{N^{*}} \beta^{i},
$$

where $N^{*}$ is the number of active atoms that participate in creating the stress in the lattice. The value of $N^{*}$ will determine the volume in which stress is averaged. It can include a few atoms to obtain a local stress value or encompass all the atoms in the system to provide a macroscopic average stress. More details of the calculation of stresses in atomistic simulations can be found in [43].

\subsection{Simulation strategy}

Void growth was analyzed by means of MD in a 2D periodic voided crystal, in which the circular voids are located at the corners of a square array (figure 1). Due to the symmetries of the problem, the MD simulations were carried out in a representative element unit cell of the voided crystal with periodic boundary conditions. The void volume fraction was held constant and equal to $10 \%$ and the length $L$ of the unit cell was changed accordingly to accommodate voids with an initial radius in the range $1.5 \mathrm{~nm}<R<100 \mathrm{~nm}$. This void volume fraction was selected to allow the direct comparison of our results with the 2D DD simulations of Segurado and LLorca [20,21]. Nevertheless, a few simulations were carried out with an initial void volume fraction of $4 \%$ to demonstrate that the result were independent of this parameter. The maximum void radius was one order of magnitude longer than those attained previously by means of 3D MD simulations $[6,26,44]$ and it was similar to the minimum size studied using 2D DD $[20,21]$. 


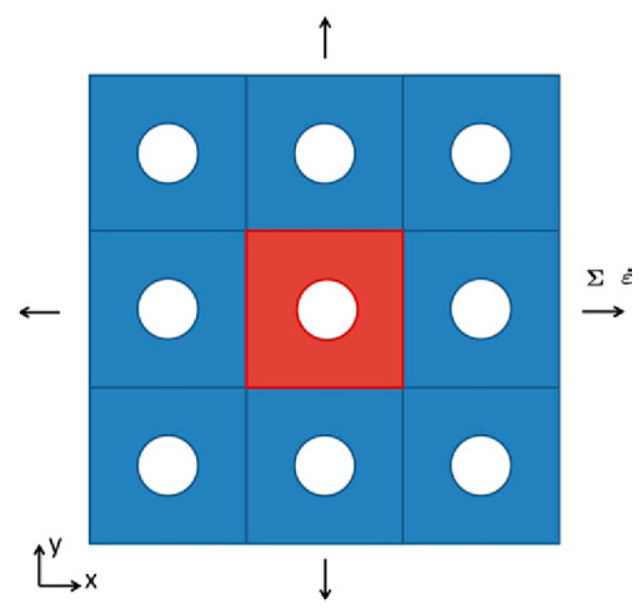

Figure 1. Periodic arrangement of the crystal with general applied far-field stress $\Sigma$ and strain rate $\dot{\varepsilon}$ fields.

(a)

(b)

(c)

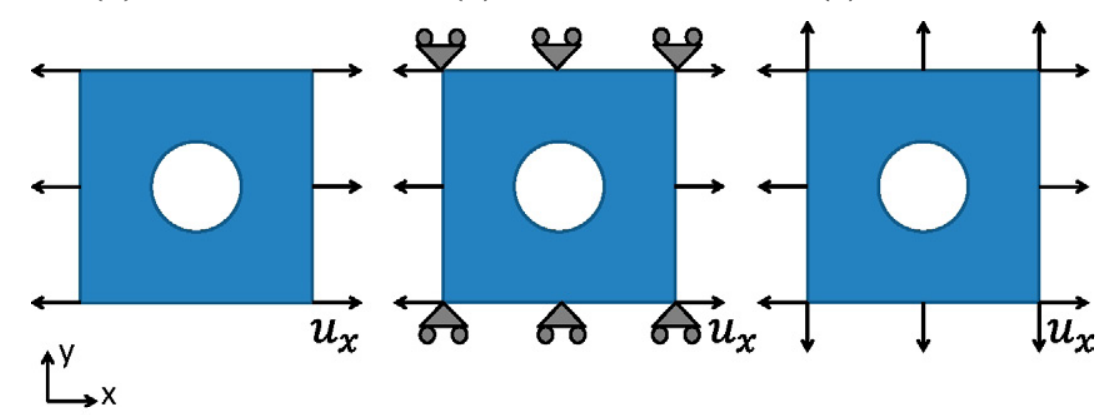

Figure 2. Loading cases considered to study void growth. (a) Uniaxial tension, $(b)$ Uniaxial deformation and $(c)$ Biaxial deformation.

Simulations were carried out assuming periodicity with three different boundary conditions, namely uniaxial tension, uniaxial deformation and biaxial deformation, which lead to very different values of multiaxiality. The corresponding boundary conditions are depicted in figure 2 . Uniaxial tension was obtained by applying a constant velocity $\pm \dot{u}_{x} / 2$ along the $x$-axis at both ends $(x=0, L)$ of the unit cell while the perpendicular boundaries were stress free $\left(\Sigma_{y y}=0\right.$ at $\left.y=0, L\right)$. Uniaxial deformation was obtained with the same velocity field at $x=0, L$ while perpendicular deformation was constrained $\left(\dot{u}_{y}=0\right.$ at $\left.y=0, L\right)$. Finally, biaxial deformation was achieved by applying the same velocity field perpendicular to the boundaries along the $x$ and $y$ directions. In all cases, dislocations leaving one external boundary of the unit cell were assumed to enter the unit cell by the opposite boundary. Simulations were carried out at strain rates $\dot{\varepsilon}\left(=\dot{u}_{x} / L\right)$ in the range $10^{7}$ to $4.0 \times 10^{8} \mathrm{~s}^{-1}$ and three different temperatures $(T=20,400$ and $800 \mathrm{~K})$. These strain rates are lower than the standard ones in MD simulations ${ }^{4}$ but they are still several orders of magnitude higher than those used in DD $\left(\approx 10^{3}-10^{4} \mathrm{~s}^{-1}\right)$.

${ }^{4}$ Although there are 3D MD simulations performed at lower strain rates of $10^{6} \mathrm{~s}^{-1}[45]$. 
In addition to the 2D simulations, a 3D model with a cylindrical void was generated to compare with the $2 \mathrm{D}$ results and analyze the effect of dimensionality. The $3 \mathrm{D}$ simulations were carried out under the standard EAM formalism for Au [46], using a simulation cell with periodic boundary conditions in all three directions. No angular term was used in the 3D case to stabilize the potential. The size of the simulation cell was $28 \times 28 \times 6.36 \mathrm{~nm}^{3}$, and the axis of the cylindrical void was parallel to the $z$-axis, which corresponds to the $\left\langle\begin{array}{llll}1 & 1 & 1\end{array}\right\rangle$ crystallographic direction. The void radius and volume fraction were $5 \mathrm{~nm}$ and $10 \%$, respectively, and simulations were performed at different temperatures up to $0.5 T_{\mathrm{m}}$, where $T_{\mathrm{m}}$ is the melting temperature. The strain rate was $10^{9} \mathrm{~s}^{-1}$. The $3 \mathrm{D}$ cell was deformed along the $x$-axis (corresponding to the $\langle 110\rangle$ crystallographic direction), allowing the simulation cell to relax along the two other directions. In order to make a direct comparison, 2D simulations were carried out using the same parameters (geometry, strain rate and temperature).

\section{Results and discussion}

\subsection{Mechanisms of plastic deformation and void growth}

Preliminary simulations were carried out to analyze the mechanisms of plastic deformation and of void growth in the 2D material. The 2D lattice was constructed as the close-packed plane to represent a general (1 111$)$ fcc or $(0001)$ hcp metal. Hexagonal packing is the most stable and dense 2D solid. It also shows (in contrast to a square lattice) a larger number of slip systems and thus a larger ductility was expected. It presents three slip systems along the three compact directions oriented at $0^{\circ}, 60^{\circ}$ and $-60^{\circ}$ from the $x$-axis. The shear modulus was of the $2 \mathrm{D}$ solid was $46.8 \mathrm{GPa}$ and the Burgers vector $2.8 \mathrm{~nm}$. Upon deformation, plastic deformation developed by the nucleation at the void surface of pure edge dislocations with Burgers vectors parallel to the compact directions. The dislocations were nucleated at atomic steps on the void surface and glided toward the interior of the material. As a result of the emission of the first dislocation, the surface step disappeared leaving behind a straight (not kinked) segment on the void perimeter (figure 3 ). This mechanism of heterogeneous dislocation nucleation from surface steps both relieves elastic stresses and reduces the surface energy of the void. Further dislocation nucleation events lead to the formation of kinks and the initial stepped perimeter (with atomic steps) of the circular void is transformed into a polygonal perimeter with ledges (multiple atomic steps), as shown in figure 3. It should be noted that stepped surfaces have been reported as preferential sites for dislocation nucleation during nanoindentation of gold surfaces and the results were confirmed by atomistic simulations $[47,48]$. In addition, void growth by heterogeneous nucleation of dislocations at the void surface has been observed experimentally under very high strain rate deformation $[4,5]$. For the sake of comparison, the dislocation nucleation in the cylindrical void simulated in $3 \mathrm{D}$ is shown in figure $3(d)$. Dislocation nucleation in 3D occurs by the formation of a loop, instead of a straight dislocation. Nevertheless, it should be noted that the mechanisms of dislocation nucleation and propagation into the bulk in 2D are in agreement with those expected for a fcc metal although in 2D. This supports the validity of our $2 \mathrm{D}$ approach to simulate plastic void growth.

\subsection{Influence of dimensionality}

As indicated in the introduction, 2D MD simulations introduce a number of simplifications and it is important to assess their influence on the results. To this end, two tests were carried out. The first one was focused in the predictions of the evolution of the elastic modulus with temperature using the $2 \mathrm{D} \mathrm{MD}$ model. This parameter was chosen because there is a large body 
(a)

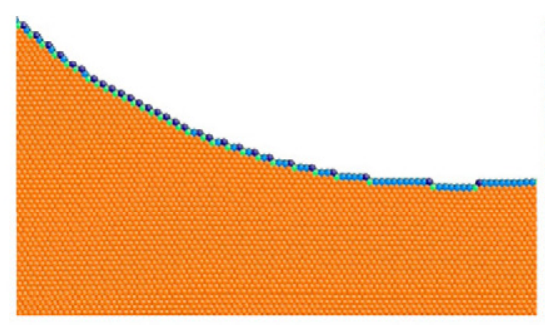

(c)

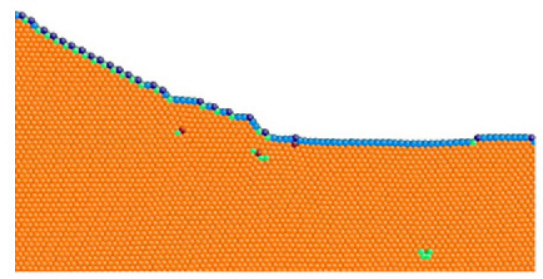

(b)

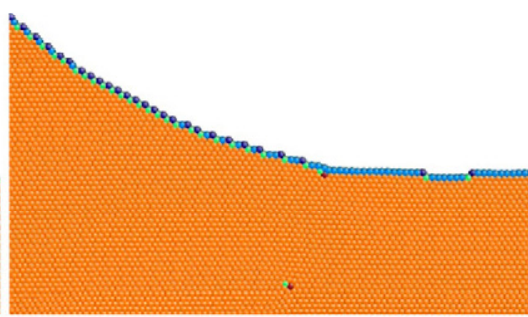

(d)

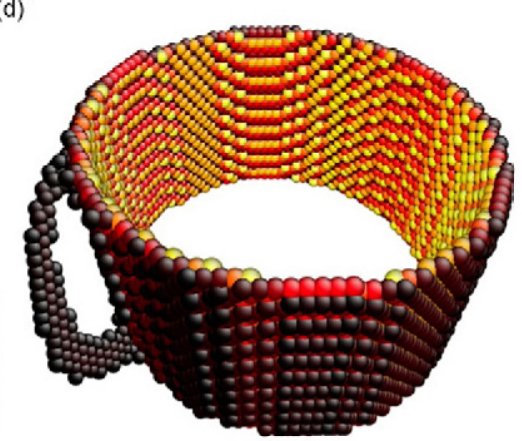

Figure 3. Nucleation of dislocations at the void surface. (a) Initial void perimeter showing the atomic steps. (b) Nucleation of dislocation leaving behind a straight segment. (c) Further emission of dislocations leading to the formation of ledges (multiple atomic steps) in the void perimeter. Atom color depends on its coordination number. $(d)$ Dislocation half-loop emerging from the surface of a 3D cylindrical void at $200 \mathrm{~K}$. Only atoms at the surface of the void and at the dislocation core are shown.

of experimental data in the literature to compare with. The results of the 2D MD simulations (normalized by the elastic modulus at $300 \mathrm{~K}$ ) are plotted in figure $4(a)$, together with the experimental data from a number of metallic alloys [49]. The 2D numerical simulations provide sensible results that fall within the range of experimental data.

The second test, more relevant to this investigation, was to explore the influence of the temperature on the stress necessary to nucleate dislocations from the surface of a cylindrical void. 2D and 3D simulations were carried out for a cylindrical (circular) void of $5 \mathrm{~nm}$ in radius in 3D (2D). The void volume fraction in the cell was $10 \%$. The predictions of the evolution of the yield stress ${ }^{5}$ (normalized by the yield stress at $0 \mathrm{~K}$ ) obtained by 2D MD and 3D MD are plotted as a function of temperature (normalized by the melting temperature, $T_{\mathrm{m}}$ ) in figure $4(b)$. Both models (2D and 3D) predicted similar trends but the reduction in yield stress with temperature was more marked in $2 \mathrm{D}$. Dislocation nucleation from the void surface requires the formation of a critical loop that has some lateral extent (along the length of the cylinder) (figure $3(d)$ ). Obviously, the 'loop' does not exist in 2D, but it can be considered to have a very short length for purposes of approximation. The energy barrier will scale with the loop size, and so the 2D barrier is much smaller, leading to a much greater reduction in the stress necessary to nucleate a dislocation with temperature (or strain rate) than in the $3 \mathrm{D}$

\footnotetext{
5 In the context of these MD simulations, the yield stress is defined as the component of the macroscopic stress tensor in the loading direction when the first stable dislocation appeared, i.e., it was emitted from the void surface and did not return back.
} 

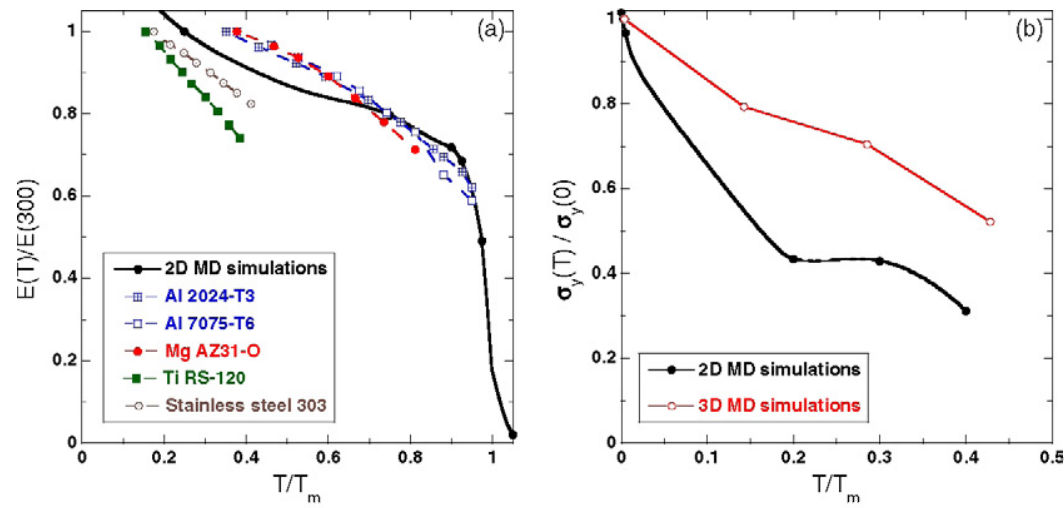

Figure 4. (a) Effect of temperature on the elastic modulus from 2D MD simulations. Experimental data for a number of metallic alloys [49] are include for comparison. The moduli are normalized by the corresponding modulus at $300 \mathrm{~K}$ and the temperatures, $T$, by the melting temperature, $T_{\mathrm{m}}$. (b) Evolution of the yield stress, $\sigma_{y}$, in a voided cell (normalized by the yield stress at $0 \mathrm{~K}$ ) as a function of temperature, $T$ (normalized by the melting temperature, $T_{\mathrm{m}}$ ) as predicted by $2 \mathrm{D}$ and 3D MD simulations.
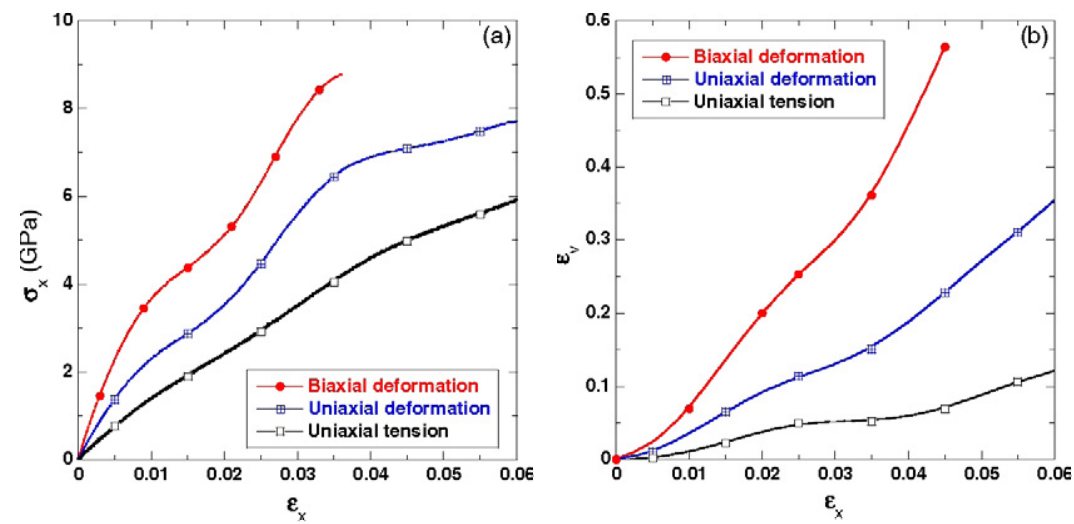

Figure 5. Influence of multiaxiality on $(a)$ stress-strain curve and $(b)$ void growth in a periodic voided crystal. The initial void volume fraction was $10 \%$, the initial void radius $30 \mathrm{~nm}$ and $T=400 \mathrm{~K}$.

system. These limitations of $2 \mathrm{D} \mathrm{MD}$ have to be accounted for to interpret the results presented below.

\subsection{Effect of hydrostatic stresses and temperature on void growth}

The influence of hydrostatic stresses and temperature on the void growth rate was studied for a representative unit cell containing a void of $30 \mathrm{~nm}$ in radius. The void volume fraction was $10 \%$ and the simulations included approximately $3.6 \times 10^{5}$ atoms. The applied far-field strain rate was $\dot{\epsilon}=4.0 \times 10^{8} \mathrm{~s}^{-1}$. Simulations to analyze the effect of hydrostatic stresses were carried out at $T=400 \mathrm{~K}$.

The effect of hydrostatic stresses on the mechanisms of plastic deformation and void growth was examined under three different loading conditions, namely uniaxial stress, uniaxial deformation and biaxial deformation. The corresponding stress-strain curves along the $x$-axis are plotted in figure 5(a). The curves present an initial elastic regime (slightly non-linear) up 
to a strain $\epsilon_{x} \approx 0.01$ and the apparent stiffness along the $x$-axis increased with the constraint. Void coalescence began at applied strains above $6 \%$ and thus the yield stress and the post-yield behavior were analyzed before this point.

After the nucleation of the first dislocation, elastic stresses around the void were relaxed and it was necessary to increase the far-field stress to nucleate new dislocations. Moreover, hardening after yielding was due to the impossibility of accommodating the imposed strain rate with plastic deformation. As shown in figure 5(a), both the yield stress and the hardening after yielding increased with the constraint. It should be mentioned that the undulations in the stress-strain curves after yielding are dynamic artifacts induced by the elastic stress waves.

The actual area of the void was computed numerically from the positions of the atoms at the void surface. To this end, the void area was discretized in small triangular sectors defined by two neighbor atoms on the void surface and a fixed point near to the void center. The void area was computed by adding the areas of all triangles.

The increase in void area divided by the initial void area, $\epsilon_{v}=\left(A_{v}-A_{v \text {,ini }}\right) / A_{v \text {,ini }}$, was plotted as a function of the applied strain in figure $5(b)$. This figure shows that multiaxiality did play a very significant role in void growth rate. Void growth under biaxial deformation was approximately two times faster than under uniaxial tension, which in turn was almost three times faster than under uniaxial tension. This behavior can be readily explained because the total imposed volumetric strain $\left(\epsilon_{x}+\epsilon_{y}\right)$ under uniaxial and biaxial deformation has to be accommodated by the elastic volumetric strain and void growth as dislocation slip only induces shear strains. As the volumetric elastic strain is always small, the higher the imposed volumetric strains, the higher the void growth rate $[24,50]$. Similar results were reported in the case of larger voided crystals (void radius in the range 80 to $500 \mathrm{~nm}$ ) in which void growth during plastic deformation was analyzed by means of 2D DD. Void growth rates are not plotted beyond $6 \%$ in figure 5 (neither the corresponding stress-strain curves) because void growth at larger strains was controlled by the coalescence of the central void with small voids nucleated in the central section of the unit cell by dislocation interactions, leading to the final fracture of the unit cell by the formation of a crack perpendicular to the loading axis.

The preferential sites for dislocation nucleation on the void perimeter depended on the stress state. Under uniaxial tension, dislocations were nucleated at void surface locations perpendicular to the loading axis (figure $6(a)$ ) on slip systems oriented at $\pm 60^{\circ}$ with the $x$-axis. The preferential nucleation sites extended toward the void poles under uniaxial deformation (figure $6(b)$ ) and dislocation nucleation occurred along the whole void perimeter and in three available slip systems under biaxial deformation (figure $6(c)$ ). These differences led to changes in the void shape with constraint as the applied strain increased, as shown in figure 7. Void growth under uniaxial tension led to an elongation of the void along the loading axis while the radius was increased uniformly under biaxial deformation. Note that the increase of void under uniaxial tension up to $\epsilon_{x}=5 \%$ was mainly due by elastic void growth as the number of dislocations nucleated from the void surface was limited.

The influence of the temperature on the void growth rate was studied at $T=20,400$ and $800 \mathrm{~K}$. The applied far-field strain rate was $\dot{\epsilon}=4 \times 10^{8} \mathrm{~s}^{-1}$ and simulations were carried out in periodic voided cells with a void radius of $30 \mathrm{~nm}$ and an initial void volume fraction of $10 \%$. The stress-strain curves of the periodic voided crystal at $T=20,400$ and $800 \mathrm{~K}$ are plotted under uniaxial tension and biaxial deformation in figures $8(a)$, respectively. The slope of the initial elastic regime slightly decreased with the temperature, as it has been reported in many materials [49,51-54]. The yield stress (marked with arrows at 400 and $800 \mathrm{~K}$ ) also decreased with temperature because yielding occurred by the nucleation of dislocations from the void surface and this mechanism is thermally activated [55]. In addition, the hardening after yielding also decreased with temperature as a result of the lower elastic modulus. 

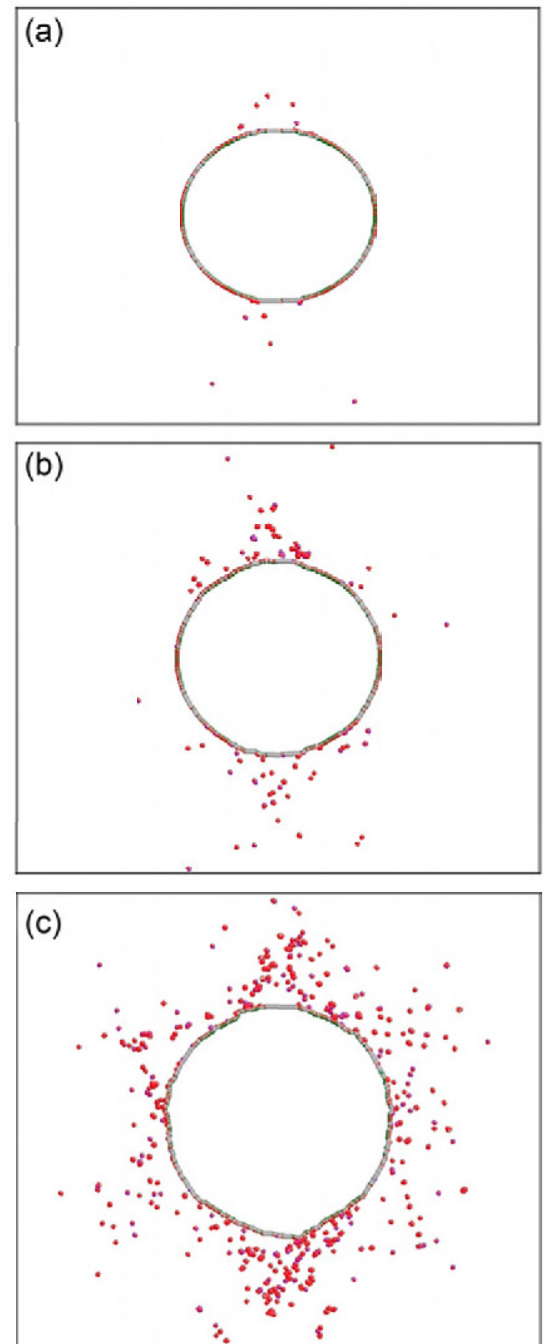

Figure 6. Influence of multiaxiality on the dislocation density around the void perimeter (a) Uniaxial tension. (b) Uniaxial deformation. (c) Biaxial deformation. $x$-axis is horizontal. Only atoms with a coordination number different than 6 (corresponding to free surfaces and dislocations) are plotted. The far-field applied strain along the $x$-axis was $5 \%$ in all cases.

The corresponding void growth rates at different temperatures are plotted in figures $8(b)$ under uniaxial tension and biaxial deformation, respectively. The void growth rate in the elastic regime was equivalent for the three temperatures while voids grew faster in the plastic regime at higher temperatures. Differences were only significant under biaxial deformation and these results indicated that the influence of temperature on the void growth rate was minor as compared with that of the hydrostatic stresses (see figure $5(b)$ ).

\subsection{Size effects on void growth}

The influence of void size on the void growth rates was studied by carrying out 2D MD simulations of periodic voided crystals with void radius in the range 5 to $50 \mathrm{~nm}$. The initial 


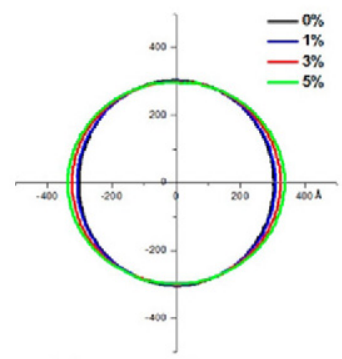

(a) Uniaxial Tension

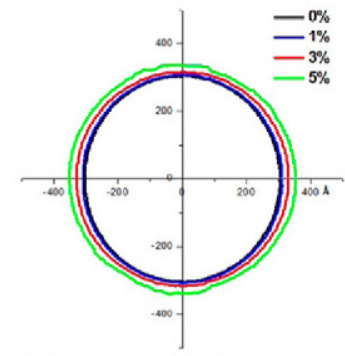

(b) Uniaxial Deformation

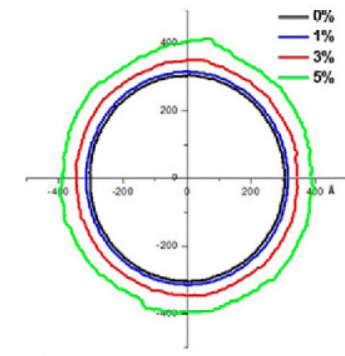

(c) Biaxial Deformation

Figure 7. Influence of multiaxiality on the void shape as a function of the applied strain (from $0 \%$ to $5 \%$ ) for periodic voided crystals subjected to different loading conditions. (a) Uniaxial tension. (b) Uniaxial deformation. (c) Biaxial deformation. $x$-axis is horizontal. Void dimensions are given in $\AA$.
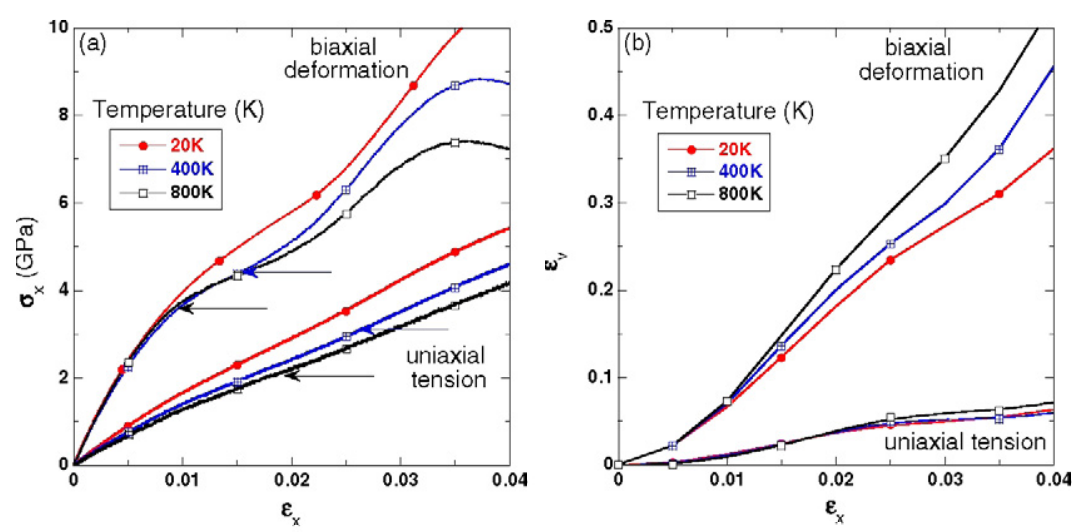

Figure 8. (a) Influence of the temperature on the stress-strain curve of the periodic voided crystal under uniaxial tension and biaxial deformation. The initial void volume fraction was $10 \%$ and the initial void radius $30 \mathrm{~nm}$. The arrows indicate the yield stress at $400 \mathrm{~K}$ (blue) and $800 \mathrm{~K}$ (black). The yield stresses at $20 \mathrm{~K}$ were much higher and are not included in the figure. $(b)$ Influence of the temperature on the void growth under uniaxial tension and biaxial deformation. The initial void volume fraction was $10 \%$ and the initial void radius $30 \mathrm{~nm}$.

void volume fraction was always equal to $10 \%$ and The crystal was loaded under biaxial deformation because growth rates are maxima under this condition and size effects should be more evident. The corresponding stress-strain curves along the $x$-axis are plotted in figure $9(a)$. They are practically superposed and the only significant difference was the superposition of undulations in the curves whose wavelength decreased with the cell size. As indicated above, this fluctuation in the curves is a dynamic effect induced by the reflection of the stress waves in the periodic boundaries. The increase in void area divided by the initial void area, $\epsilon_{\mathrm{v}}$, was plotted as a function of the applied strain in figure $9(b)$. No appreciable size effect was detected for void radius in the range 15 to $50 \mathrm{~nm}$ but smaller voids seemed to grow slightly faster when $R<10 \mathrm{~nm}$. In all cases, the mechanisms of plastic deformation was equivalent: nucleation of dislocations from the void surface, followed by dislocation slip toward the interior of the crystal.

Previous MD studies by [26] reported a size effect in void growth of the type 'smaller is faster' and a similar effect also was found in our 2D MD simulations when the loading rate was higher than $10^{9} \mathrm{~s}^{-1}$ but not at lower loading rates. This size effect may be an artifact as we 

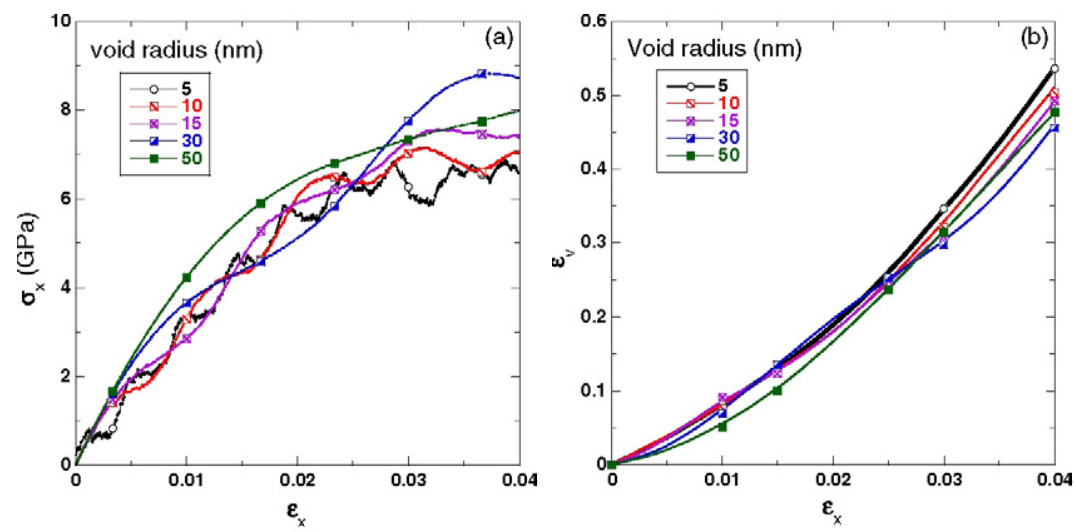

Figure 9. Influence of void radius on $(a)$ stress-strain curve and $(b)$ void growth in a periodic voided crystal loaded under biaxial deformation at $\dot{\epsilon}=4.0 \times 10^{8} \mathrm{~s}^{-1}$ and $T=400 \mathrm{~K}$. The initial void volume fraction was $10 \%$.

found that it only appeared when the applied velocity at the cell boundaries (which depends on strain rate and cell size) was higher than the elastic wave speed. This was the case in [26] $\left(\dot{\epsilon}=10^{10} \mathrm{~s}^{-1}\right)$ and in our simulations for strain rates equal to or higher than $10^{9} \mathrm{~s}^{-1}$. Under these conditions, the strain energy is accumulated at the cell boundary and the strain energy near void is always lower than that at void boundaries, in opposition to the standard behavior under quasi-static equilibrium. This effect is enhanced by the large cell size and leads to the apparent size effect the smaller is faster. This apparent effect disappears if the applied velocity on cell surfaces becomes small as compared to elastic wave speed.

In addition, it is also worth noting that 2D DD simulations of voided crystals have reported a very strong size effect in the stress-strain curve ('smaller is stronger') and in the void growth rates ('smaller is slower') [20]. The differences in the size effects have to be related to the corresponding mechanisms of plastic deformation, as detailed below. In the case of large voids $(R>100 \mathrm{~nm})$ and for typical values of the density of dislocation sources in the bulk $\left(\approx 100 \mu \mathrm{m}^{-2}\right)$ [21], plastic deformation in voided crystals is always dominated the nucleation of dislocations from the bulk, which occurs at much lower stresses than those necessary to nucleate dislocations from the void surface. Of course, the larger the crystal, the higher the number of available sources to promote plastic deformation and larger crystals can accommodate more easily the applied strain by plastic slip. However, as the crystal size (and the number of sources) decreases, the applied strain has to be partially accommodated by elastic deformation, leading to a marked hardening after yielding and to the size effect 'smaller is stronger' found in 2D DD simulations. Similarly, void growth by plastic deformation is inhibited in smaller crystals, leading to the 'smaller is slower' size effect in void growth [20].

In the case of very small voids $(R<50 \mathrm{~nm})$, there are not many sources available in the voided crystal whose size is much smaller than the average distance between sources $(0.1 \mu \mathrm{m}$ for dislocation source densities of $100 \mu \mathrm{m}^{-2}$ ). As a result, plastic deformation is controlled by dislocation nucleation at the void surface at much higher stresses. The sources available in this case are proportional to the void radius and the increase in void area induced by each dislocation is also proportional to the void radius. Thus, the total increase in void area due to geometrical effects should be proportional to $R^{2}$, which divided by the initial void area $\pi R^{2}$ leads to the absence of a size effect in void growth, in agreement with the 2D MD simulations presented above. 

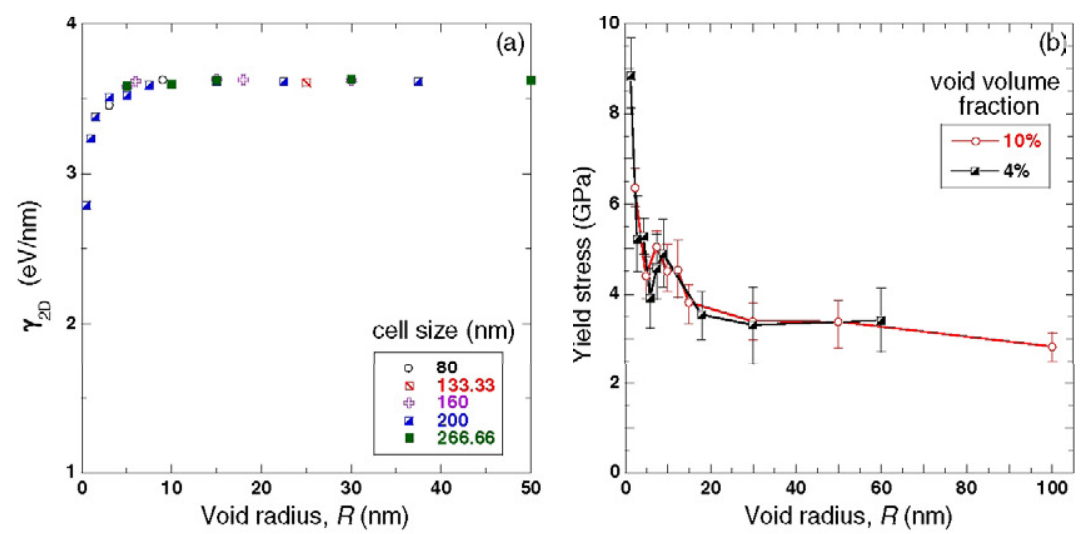

Figure 10. (a) Evolution of the energy per unit length associated at the void surface, $\gamma_{2} \mathrm{D}$, as a function of the void radius $R$ at $400 \mathrm{~K}$ for periodic unit cells of different sizes. (b) Influence of void radius (both $4 \%$ and $10 \%$ of volume fraction) on the yield strength at $400 \mathrm{~K}$. These results were obtained from 2D MD simulations with $\dot{\epsilon}=8 \times 10^{7} \mathrm{~s}^{-1}$. Average values and standard deviations of the yield stress were obtained from uncertainty in the stress at which the dislocation was emitted because the position of atoms to determine the first emitted dislocation are saved every 500 simulation steps.

This line of reasoning fails in the case of extremely small voids ( $R<10 \mathrm{~nm}$ ), when the surface energy per unit length at the void perimeter becomes a significant factor because of the small void curvature. The surface energy associated with the void depends upon the number of missing neighbors for the atoms at the void surface. This value decreases with the void radius because of the smaller radius of curvature and converges rapidly toward the surface energy of a straight surface when the void radius increases. Several methods are available to determine the surface energy in MD simulations [56-58]. In our 2D study, the energy per unit length associated to the void surface, $\gamma_{2}$, can be computed as the difference in the total energy in a voided cell, $E_{\mathrm{v}}$, and in an unvoided cell, $E_{\mathrm{u}}$, where $E_{\mathrm{u}}$ was normalized by the ratio between number of atoms of unvoided cell $\left(N_{\mathrm{u}}\right)$ and voided cell $\left(N_{\mathrm{v}}\right)$,

$$
\gamma=\frac{E_{\mathrm{v}}-\frac{N_{\mathrm{v}}}{N_{\mathrm{u}}} E_{\mathrm{u}}}{2 \pi R},
$$

where $E_{\mathrm{v}}$ and $E_{\mathrm{u}}$ are obtained from the total potential energy of the voided and unvoided cell, respectively, and $N_{\mathrm{v}}$ and $N_{\mathrm{u}}$ are the total number of atoms in the corresponding cells. The total potential energy of voided and unvoided cells of different size (from 80 to $266.6 \mathrm{~nm}$ ) was computed at $400 \mathrm{~K}$ using $2 \mathrm{D} \mathrm{MD}$ and $\gamma_{2} \mathrm{D}$ is plotted as a function of void radius $R$ in figure $10(a)$. These results clearly show that the surface energy becomes important when the void radius is below $5 \mathrm{~nm}$.

Thus, for very small voids $(R<10 \mathrm{~nm})$, void surfaces become more stable and higher stresses are necessary to nucleate dislocations from the void surface. This is demonstrated in figure $10(b)$, in which the yield stress (understood as the far-field applied stress necessary to nucleate the first stable dislocation) is plotted as a function of the void radius. These results were obtained with 2D MD simulations under biaxial deformation at $400 \mathrm{~K}$ and an applied strain rate $\dot{\epsilon}=8 \times 10^{7} \mathrm{~s}^{-1}$ for voids in the range 1.5 to $100 \mathrm{~nm}$. Two different initial void volume fractions (4\% and 10\%) were considered. The yield stress increased rapidly as the void radius became smaller than $10 \mathrm{~nm}$ in agreement with the increase in the surface energy density in figure $10(a)$. In addition, this behavior is consistent with the 2D nucleation model 


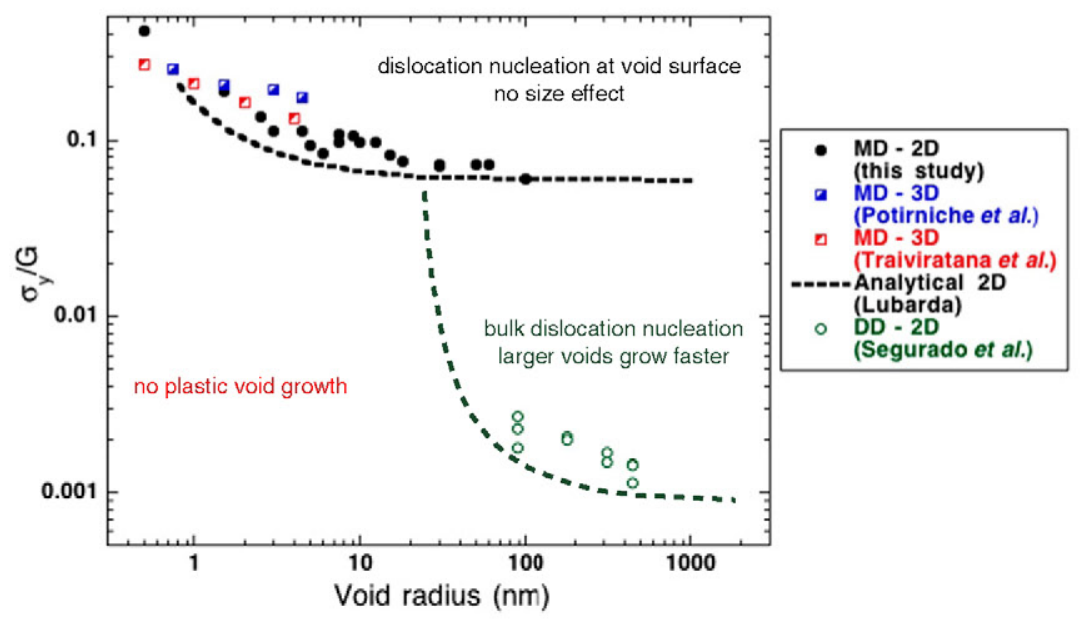

Figure 11. Mechanisms of void growth by plastic deformation as a function of void radius.

presented in [23] that predicts an increase in the stress necessary to nucleate a dislocation from void surface when void radius is below $10 \mathrm{~nm}$. The results were independent of the volume fraction, as expected.

These results can be discussed in combination with previous simulations of void growth obtained using 3D MD [26,27], the 2D theoretical model of Lubarda model presented in [23] as well as 2D DD $[20,21]$ to have a wider view on the mechanisms of void growth and the corresponding size effects for void radius in the range of nm to $\mu \mathrm{m}$. The yield stress, $\sigma_{y}$ (understood as the stress in the loading direction necessary to nucleate the first stable dislocation either from the bulk or from the void surface) normalized by the shear modulus $G$ is plotted as a function of the void radius in figure 10 according Potirniche et al [26], Traiviratana et al [27], Lubarda [23], Segurado and LLorca [20,21] and the results of the present study. This plot shows three different regions for void growth driven by mechanical stresses under the assumption that diffusion effects are negligible. For large voids, $(R>100 \mathrm{~nm}), 2 \mathrm{D}$ DD simulations [20] that plastic void growth occurs by the nucleation of dislocations in the bulk at stresses below $0.01 \mathrm{G}$. In this region, larger voids grow faster than smaller ones because the larger availability of dislocation nucleation sources around the void increases the number of dislocations that escape the material through the void surface and contribute to the void growth. This mechanism is exhausted as the void radius decreases for typical values of the dislocation densities $\left(10^{12}-10^{14} \mathrm{~m}^{-2}\right)$, leading to a region in which plastic void growth is not possible when $R<50 \mathrm{~nm}$ and $\sigma_{y} / G<0.05$. But even very small voids can grow at very high stresses $\left(\sigma_{y} / G \approx 0.1\right)$ by the nucleation of dislocations at the void surface. This regime is only found at very high strain rates [8] and, as opposed to void growth by dislocation nucleation in the bulk, void growth rates are independent of the void radius.

It is interesting to note that the results for the yield stress of our 2D MD simulations are in close agreement with the 2D analytical model suggested by Lubarda [23]. Moreover, recent 3D MD simulations of Tang et al also indicated that the stress necessary to nucleate dislocations from the void surface was independent of the void radius. The yield stress reported in this study was lower $\left(0.01<\sigma_{y} / G<0.1\right)$ but the differences came from the definition of the yield stress because Tang et al defined yield stress as the stress necessary to nucleate a defect in the void surface which is much lower than stress necessary to emit a stable dislocation. 


\section{Conclusions}

The mechanisms of void growth by plastic deformation were studied in a two-dimensional framework by means of molecular dynamics of a 2D periodic voided crystal, in which the circular voids are located at the corners of a square array. To this end, a suitable interatomic potential was adapted to obtain a $2 \mathrm{D}$ material whose behavior was in agreement with the standard results for dislocation plasticity in fcc metals. In the range of void sizes analyzed (1.5 to $100 \mathrm{~nm}$ ), it was found that plastic deformation was triggered by the nucleation of dislocations at the atomic steps of the void surfaces. The yield stress, defined as the stress necessary to nucleate stable dislocations, was independent of the void radius except for very small voids $(R<10 \mathrm{~nm})$, in which the reduction in the surface energy density associated to the void surface increased the yield stress. The yield stress and the hardening rate after yielding of the periodic voided crystal decreased with temperature but the void growth rate was not very sensitive to this factor compared with multiaxiality effect. Simulations under uniaxial tension, uniaxial deformation and biaxial deformation showed that the void growth rate increased very rapidly with the hydrostatic stresses.

Comparison of these results with previous 3D molecular dynamics simulations of very small voids $(R<$ order of $10 \mathrm{~nm})$ and with $2 \mathrm{D}$ dislocation dynamics simulations of larger voids $(R>100 \mathrm{~nm})$ showed three different regions for void growth driven by mechanical stresses in absence of diffusion. Void growth in large voids $(R>100 \mathrm{~nm})$ occurs at stresses below $0.01 G$ by nucleation of dislocations in the bulk and is associated to a size effect of the type 'larger is faster'. Small voids $(R<50 \mathrm{~nm})$ can only grow by nucleation of dislocations at the void surface at very high stresses $(\approx 0.1 \mathrm{G})$, and there is not size effect in void growth under these conditions. At lower stresses, these small voids can only grow by diffusion.

\section{Acknowledgments}

This investigation was supported by the Spanish Ministry of Economy and Competitiveness through grants MAT2009-14396 and MAT2009-14578-C03-02. HJC acknowledges the support from the Moncloa International Campus of Excellence through the PICATA program.

\section{References}

[1] Donnelly S E 1985 The density and pressure of helium in bubbles in implanted metals: a critical review Radiat. Effects 90 1-47

[2] Babout L, Maire E and Fougeres R 2004 Damage initiation in model metallic materials: X-ray tomography and modelling Acta Mater. 52 2475-87

[3] Moriarty J A, Belak J F, Söderlind P, Rudd R E and Streitzand F H and Yang L H 2002 Quantum-based atomistic simulation of materials properties in transition metals J. Phys.: Condens. Matter $142825-57$

[4] Stevens A L, Lee Davison and Warren W E 1972 Spall fracture in aluminum monocrystals: a dislocationdynamics approach J. Appl. Phys. 43 4922-7

[5] Lubarda V A, Schneider M S, Kalantar D H, Remington B A and Meyers M A 2004 Void growth by dislocation emission Acta Mater: 52 1397-408

[6] Ahn D C, Sofronis P and Minich R 2006 On the micromechanics of void growth by prismatic-dislocation loop emission J. Mech. Phys. Solids 54 735-55

[7] Bulatov V V, Wolfer W G and Kumar M 2010 Shear impossibility: Comments on 'Void growth by dislocation emission and void growth in metals: atomistic calculations' Scr. Mater. 63 144-7

[8] Nguyen L D and Warner D H 2012 Improbability of void growth in aluminum via dislocation nucleation under typical laboratory conditions Phys. Rev. Lett. 108035501

[9] Benzerga A and Leblond J-B 2010 Ductile fracture by void growth to coalescence Adv. Appl. Mech. 44 169-305

[10] Fleck N A, Muller G M, Ashby MF and Hutchinson J W 1994 Strain gradient plasticity: Theory and experiment Acta Metall. Mater. 42 457-87 
[11] Nix W D 1997 Elastic and plastic properties of thin films on substrates: nanoindentation techniques Mater. Sci. Eng. A 234 37-44

[12] Uchic M D, Shade P A and Dimiduk D M 2009 Plasticity of micrometer-scale single crystals in compression Annu. Rev. Mater. Res. 39 361-86

[13] Shu J Y 1998 Scale-dependent deformation of porous single crystals Int. J. Plast. 14 1085-107

[14] Liu B, Qiu X, Huang Y, Hwang K C, Li M and Liu C 2003 The size effect on void growth in ductile materials J. Mech. Phys. Solids $\mathbf{5 1} 1171-87$

[15] Liu B, Huang Y, Li M, Hwang K C and Liu C 2005 A study of the void size effect based on the Taylor dislocation model Int. J. Plast. 21 2107-22

[16] Borg U and Kysar J W 2007 Strain gradient crystal plasticity analysis of a single crystal containing a cylindrical void Int. J. Solids Struct. $446382-97$

[17] Borg U, Niordson C F and Kysar J W 2008 Size effects on void growth in single crystals with distributes voids Int. J. Plast. 24 688-701

[18] Huang M, Li Z and Wang C 2007 Discrete dislocation dynamics modelling of microvoid growth and its intrinsic mechanism in single crystals Acta Mater. 55 1387-96

[19] Hussein M, Borg U, Niordson C F and Deshpande V S 2008 Plasticity size effects in voided crystals $J$. Mech. Phys. Solids $56114-31$

[20] Segurado J and LLorca J 2009 An analysis of the size effect on void growth in single crystals using discrete dislocation dynamics Acta Mater. 57 1427-36

[21] Segurado J and LLorca J 2010 Discrete dislocation dynamics analysis of the effect of lattice orientation on void growth in single crystals Int. J. Plast. 26 806-19

[22] Chang H-J, Segurado J and LLorca J 2013 Simulation of void growth by means of 3d dislocation dynamics Scr. Mater: in preparation

[23] Lubarda V A 2011 Emission of dislocations from nanovoids under combined loading Int. J. Plast. 27 181-200

[24] Seppälä E T, Belak J and Rudd R E 2004 Onset of void coalescence during dynamic fracture of ductile metals Phys. Rev. Lett. 93245503

[25] Marian J, Knap J and Ortiz M 2004 Nanovoid cavitation by dislocation emission in aluminum Phys. Rev. Lett. 93165503

[26] Potirniche G P, Horstemeyer M F, Wagner G J and Gullett P M 2006 A molecular dynamics study of void growth and coalescence in single crystal nickel Int. J. Plast. 22 257-78

[27] Traiviratana S, Bringa E M, Benson D J and Meyers M A 2008 Void growth in metals: Atomistic calculations Acta Mater. $\mathbf{5 6} 3874-86$

[28] Deng X, Zhu W, Zhang Y, He H and Jing F-Q 2010 Configuration effect on coalescence of voids in single-crystal copper under shock loading Comput. Mater. Sci. 50 234-8

[29] Deng X-L, Song Z-F, He H-L, Zhu W-J and Jing F-Q 2009 Microscopic mechanism of void coalescence under shock loading Acta Phys. Sin. 58 4772-8

[30] Zhu W, Song Z, Deng X, He H and Cheng X 2007 Lattice orientation effect on the nanovoid growth in copper under shock loading Phys. Rev. B 75024104

[31] Luo J, Zhu W, Lin L-B, He H and Jing F-Q 2005 Molecular dynamics simulation of void growth in single crystal copper under uniaxial impacting Acta Phys. Sin. 54 2791-98

[32] Tang Y, Bringa E M and Meyers M A 2012 Ductile tensile failure in metals through initiation and growth of nanosized voids Acta Mater. 60 4856-65

[33] Gungor M R and Maroudas D 2005 Relaxation of biaxial tensile strain in ultrathin metallic films: ductile void growth versus nanocrystalline domain formation Appl. Phys. Lett. 87171913

[34] van der Giessen E and Needleman A 1995 Discrete dislocation plasticity: a simple planar model Modelling Simul. Mater. Sci. Eng. 3 689-735

[35] Romero I, Segurado J and LLorca J 2008 Dislocation dynamics in non-convex domains using finite elements with embedded discontinuities Modelling Simul. Mater. Sci. Eng. 16035008

[36] Deshpande V S, Needleman A and Van der Giessen E 2005 Plasticity size effects in tension and compression of single crystals J. Mech. Phys. Solids 53 2661-91

[37] Chng A C, O'Day M P, Curtin W A, Tay A O A and Lim K M 2006 Fracture in confined thin films: a discrete dislocation study Acta Mater: 54 1017-27

[38] Widjaja A, Van der Giessen E and Needleman A 2005 Discrete dislocation modelling of submicron indentation Mater. Sci. Eng. A 400-401 456-9

[39] Segurado J, LLorca J and Romero I 2007 Computational issues in the simulation of two-dimensional discrete dislocation dynamics Modelling Simul. Mater. Sci. Eng. 15 361-75

[40] Abraham F F 1996 Dynamics of brittle fracture with variable elasticity Phys. Rev. Lett. 77869 
[41] Hatano T 2004 Dislocation nucleation in shocked FCC solids: effects of temperature and preexisting voids Phys. Rev. Lett. 93085501

[42] Ercolesi F, Parrinello M and Tosatti E 1988 Simulation of gold in the glue model Phil. Mag. A 58 213-26

[43] Zimmerman J A, Webb E B III, Hoyt J J, Jones R E, Klein P A and Bammann D J 2004 Calculation of stress in atomistic simulation Modelling Simul. Mater. Sci. Eng. 12 S319

[44] Gungor M R, Maroudas D and Zhou S J 2000 Molecular-dynamics study of the mechanism and kinetics of void growth in ductile metallic thin films Appl. Phys. Lett. 77 343-5

[45] Rudd R E 2009 Void growth in bcc metals simulated with molecular dynamics using the finnis-sinclair potential Phil. Mag. 89 3133-61

[46] Foiles S M 1985 Application of the embedded-atom method to liquid transition metals Phys. Rev. B 32 3409-15

[47] Navarro V, Rodriguez de la Fuente O, Mascaraque A and Rojo J M 2008 Uncommon dislocation processes at the incipient plasticity of stepped gold surfaces Phys. Rev. Lett. 100105504

[48] Carrasco E, de la Fuente O R, Gonzalez M A and Rojo J M 2004 Characterising and controlling surface defects Eur. Phys. J. B $40421-6$

[49] Vosteen L F 1958 Effect of temperature on dynamic modulus of elasticity of some structural alloys, National Advisory Committee for Aeronautics

[50] Zaidman M and Ponte-Castaneda P 1994 Constitutive models for porous materials with evolving microstructure J. Mech. Phys. Solids 42 1459-97

[51] Ramesh K T and Lennon A M 2004 The influence of crystal structure on the dynamic behavior of materials at high temperatures Int. J. Plast. 20 269-90

[52] Cohen R, Wasserman E and Stixrude L 1996 Thermal properties of iron at high pressures and temperatures Phys. Rev. B 53 8296-309

[53] Govaert L, Brown B and Smith P 1992 Temperature dependence of the young's modulus of oriented polyethylene Macromolecules 25 3480-3

[54] Lee S-H, Jeong J-H, Chung S-H and Kwon D 2003 Evaluation of elastic properties and temperature effects in Si thin films using an electrostatic microresonator J. Microelectromech. Syst. 12 524-30

[55] Zhu T, Li J, Samantaand A, Leach A and Gall K 2008 Temperature and strain-rate dependence of surface dislocation nucleation Phys. Rev. Lett. 100025502

[56] Zhang J-M, Ma F and Xu K-W 2004 Calculation of the surface energy of FCC metals with modified embeddedatom method Appl. Surf. Sci. 229 34-42

[57] Gloor G J, Jackson G, Blas F J and de Miguel E 2005 Test-area simulation method for the direct determination of the interfacial tension of systems with continuous or discontinuous potentials J. Chem. Phys. 123134703

[58] Laird B B and Davidchack R L 2005 Direct calculation of the crystal melt interfacial free energy via molecular dynamics computer simulations J. Phys. Chem. B 109 17802-12 